\title{
Coi1-Dependent Signaling Pathway Is Not Required for Mi-1-Mediated Potato Aphid Resistance
}

\author{
Kishor K. Bhattarai, ${ }^{1}$ Qi-Guang Xie, ${ }^{1}$ Daniel Pourshalimi,, ${ }^{1}$ Ted Younglove, ${ }^{2}$ and Isgouhi Kaloshian ${ }^{1}$ \\ ${ }^{1}$ Department of Nematology, University of California, Riverside CA 92521 USA; ${ }^{2}$ Department of Statistics, \\ University of California, Riverside, CA 92521, U.S.A.
}

Submitted 28 July 2006. Accepted 15 September 2006.

\begin{abstract}
Tomato (Solanum lycopersicum) has a unique resistance gene, $M i-1$, that confers resistance to animals from distinct taxa, nematodes, and piercing and sucking insects. $\mathrm{Mi}-1$ encodes a protein with a nucleotide-binding site and leucinerich repeat motifs. Early in the potato aphid (Macrosiphum euphorbiae)-tomato interactions, aphid feeding induces the expression of the jasmonic acid (JA)-regulated proteinase inhibitor genes, Pin1 and Pin2. The jail-1 (jasmonic acid insensitive 1) tomato mutant, which is impaired in JA perception, was used to gain additional insight into the JA signaling pathway and its role in the Mi-1-mediated aphid resistance. The jail-1 mutant has a deletion in the Coil gene that encodes a putative F-box protein. In this study, aphid colonization, survival, and fecundity were compared on wild-type tomato and jail-1 mutant. In choice assays, the jail-1 mutant showed higher colonization by potato aphids compared with wild-type tomato. In contrast, nochoice assays showed no difference in potato aphid survival or fecundity between jail-1 and the wild-type parent. Plants homozygous for $\mathrm{Mi}-\mathrm{I}$ and for the jail mutation were not compromised in resistance to potato aphids, using either choice or no-choice assays. In addition, the accumulation of JA-regulated Pin1 transcripts after aphid feeding was Coil dependent. Taken together, these data indicate that, although potato aphids activate Coil-dependent defense response in tomato, this response is not required for $\mathrm{Mi}$-1mediated resistance to aphids.
\end{abstract}

Faced with continual threats from myriad biotic and abiotic agents, plants have evolved a number of strategies to help ensure their own survival. In addition to physical barriers at the tissue surface, plants fight infection using basal defenses and classical gene-for-gene resistance responses. While basal defenses do little to mitigate the growth and multiplication of pests and pathogens, they do limit disease severity. Gene-forgene resistance is mediated by plant resistance $(R)$ genes (Flor $1971)$ and is initiated via specific recognition of a pathogenderived avirulence ( $a v r$ ) gene product by a host $R$ gene product. Both basal defense and $R$ gene-mediated defense responses involve pathways regulated by plant hormones, including salicylic acid (SA), jasmonic acid (JA), and ethylene (Hammond-Kosack and Parker 2003; Martin et al. 2003).

The tomato gene $\mathrm{Mi}-\mathrm{I}$ is the first cloned insect resistance gene (Kaloshian 2004). Besides conferring resistance to potato

Corresponding author: I. Kaloshian; Telephone: +1.951.827.3913; Fax: +1.951.827.3719; E-mail: isgouhi.kaloshian@ucr.edu

Current address of Q.-G. Xie: Department of Biology, University of Tennessee, Nashville, TN, U.S.A. aphid (Macrosiphum euphorbiae Thomas), Mi-1 also confers resistance to three species of root-knot nematodes (Meloidogyne arenaria [Neal] Chitwood, M. incognita [Kofoid \& White] Chitwood, and M. javanica [Treub] Chitwood) and two biotypes of whiteflies (Bemisia tabaci Gennadius and B. tabaci biotype B) (Milligan et al. 1998; Nombela et al. 2003). Mi-1 was introgressed into cultivated tomato, Solanum lycopersicum L., from its wild relative Solanum peruvianum L. (Smith 1944). Mi-1 belongs to the largest class of resistance genes cloned to date (Martin et al. 2003). It encodes a protein with a coiled-coil domain, nucleotide-binding site, and leucine-rich repeat (LRR) motifs (Milligan et al. 1998). In addition to Mi-1, nematode and insect resistance in tomato requires the presence of another gene, Rmel. Rmel was discovered in a genetic screen and appears to be specific for Mi-1 function (Martinez de Ilarduya et al. 2001, 2004). The identity of this gene is still unknown.

Mi-1 appears to mediate distinct resistance mechanisms to nematodes and insects, or the manifestation of resistance is distinct in roots compared with leaves. $M i-1-$ mediated defense responses to nematodes are associated with induction of hypersensitive response (HR) (Dropkin 1969), one of the hallmarks of gene-for-gene resistance. In contrast, no HR is involved in $M i-1$-mediated resistance to aphids (Martinez de Ilarduya et al. 2003). Aphids maintained on Mi-1 plants are characterized by reduced feeding, fertility, and survival (Kaloshian et al. 1997, 2000). Starvation and desiccation are the likely causes of death, as symptoms subside when aphids are transferred from resistant to susceptible genotypes (Kaloshian et al. 1997).

Another distinct feature of $M i-1-$ mediated resistance to insects is that resistance is developmentally regulated, with Mi-1 tomato plants remaining susceptible to insects for up to five weeks (Kaloshian et al. 1995; Pascual et al. 2000). In adult plants, fully expanded leaves are resistant to aphids irrespective of leaf position, while expanding leaves remain aphid-susceptible throughout the life of the plant (Kaloshian et al. 1997). The developmental regulation of $M i-1$ is absent in roots, in which resistance to nematodes is expressed early in development. It is not clear whether the developmental regulation is associated with $M i-1 . M i-1$ transcripts are present in leaves and roots early in development, and transcript levels do not change after challenge with either nematode or insect (Martinez de Ilarduya and Kaloshian 2001). Therefore, either $M i-1$ is posttranscriptionally regulated differently in roots and leaves or another component in the resistance signaling pathway is developmentally regulated differentially in roots and leaves.

Similar to plant responses to pathogens, plant defense responses to piercing and sucking insects involve the accumulation of pathogenesis-related (PR) proteins and RNA (Kaloshian 
and Walling 2005; Thompson and Goggin 2006). In the tomatopotato aphid interactions, aphid infestation induces faster and higher levels of $P R-1$ transcript accumulation in $M i-1$ tomato, the incompatible interaction, compared with the compatible interaction (Martinez de Ilarduya et al. 2003). In the incompatible $M i-1$ aphid interaction, $P R-1$ transcripts were detected as early as $6 \mathrm{~h}$ and remained at high levels $48 \mathrm{~h}$ after aphid infestations. In general, $P R-1$ expression is associated with the SA signaling pathway, although in tomato, $P R-1$ transcripts have been shown to accumulate after exogenous application of SA, methyl jasmonate (MeJA), or ethylene (Chao et al. 1999; Li et al. 2006; van Kan et al. 1995). A role for the SA signaling pathway in $M i-1-$ mediated resistance to aphids was identified (Li et al. 2006). Introducing NahG, which degrades SA to catechol, in the $\mathrm{Mi}-1$ background attenuated the resistance to potato aphids. Similarly, root-knot nematode growth was observed in Agrobacterium rhizogenes transgenic roots expressing $N a h G$ in the presence of $M i-1$, indicating also a role for SA in $\mathrm{Mi}$-1-mediated resistance to root-knot nematodes (Branch et al. 2004).

The role of JA and ethylene-regulated defense pathways in Mi-1-mediated aphid resistance remains unclear. Although cross talk among SA, JA, and ethylene pathways has been documented, simultaneous activation of plant defenses responsive to these three signal molecules has only recently been documented (Schenk et al. 2000). In tomato, proteinase inhibitors Pin 1 and Pin 2 transcripts accumulated transiently $6 \mathrm{~h}$ after aphid infestation in both compatible and incompatible aphid interactions (Martinez de Ilarduya et al. 2003). Pin1 and Pin2 are regulated by JA, which is the terminal product of the octadecanoid pathway (Creelman and Mullet 1997; Farmer and Ryan 1992). Therefore, the upregulation of these genes by aphid feeding may implicate the octadecanoid signaling pathway in aphid resistance.

To investigate the role of JA in $\mathrm{Mi}-1-$ mediated resistance to potato aphids, the well-characterized tomato jasmonic acid insensitive 1 (jail-1) mutant was used ( $\mathrm{Li}$ et al. 2001). Although jail-1 mutant plants are able to synthesize JA, they are impaired in JA perception ( $\mathrm{Li}$ et al. 2001). Recently, the jail mutation was cloned and was shown to correspond to mutations in the tomato homolog of the Arabidopsis COIl gene, which encodes a protein with a putative N-terminal F-box domain and 16 imperfect LRR (Li et al. 2004; Xie et al. 1998). COI1 is a part of the SCF complex, a multiprotein complex that includes Cullin and Skp1 and functions as an E3-type ubiquitin ligase (Bai et al. 1996). It is postulated that JA is regulated by a repressor that is recruited by the LRR domain of COI1 to the E3 complex and is marked for degradation through ubiquitination by the $26 \mathrm{~S}$ proteosome (Turner et al. 2002).

The Coil-dependent defense responses to potato aphids were evaluated using the tomato jail-1 mutant and its wildtype parent. After introducing the jail mutation in the $M i-1$ background, the requirement for Coil in the $\mathrm{Mi}$-1-mediated aphid resistance was also evaluated. Our results indicated no role for the Coil-dependent responses in basal defense or $\mathrm{Mi}-1$ mediated resistance to potato aphids in tomato.

\section{RESULTS}

The role of $\mathrm{JA}$ in basal resistance to potato aphid.

To investigate whether the Coil-dependent signaling plays a role in tomato defense against aphids, the interaction of the potato aphid with jail-1 mutant tomato versus its wild-type parent tomato cv. Castlemart (CM) were compared. Since homozygous jail-1 plants are sterile ( $\mathrm{Li}$ et al. 2004), the mutation is maintained in heterozygous state. To obtain homozy- gous jail-1 plants, germinating seeds segregating for this locus were treated with MeJA. Seedlings insensitive to MeJA were identified and transplanted. The jail-1 mutant contains a 6.2$\mathrm{kb}$ deletion in the Coil gene (Li et al. 2004). The genotype of these plants was further confirmed by the presence of the deletion in the Coil gene, using multiplex polymerase chain reaction (PCR) as described by Li and associates (2004) (data not shown).

Initially, tomato plants were screened in "choice" assays in which insects were allowed to choose between the wild-type cv. CM and jail-1 mutant plants. Choice assays therefore allowed the determination of plant-specific aphid feeding preferences. Two weeks after aphid exposure, the number of aphids on the jail-1 mutant was significantly higher $(P<0.05)$ than the number of aphids on the wild-type parent (Fig. 1).

To further evaluate the role of JA in aphid resistance, "nochoice" aphid assays were used, in which aphids were confined to a single leaflet. One-day-old adult aphids were used to accurately evaluate aphid survival and avoid variation resulting from age-dependent insect responses. The survival and fecundity of aphids were monitored on a daily basis on the jail-1 mutant and the wild-type parent. Except for day 16, the percent daily aphid survival was not significantly different on jail-1 mutant plants compared with cv. CM plants $(P>0.05)$ (Fig. 2A). On day 16, a significant difference $(P<0.01)$ in aphid survival was noted because one aphid survived on the jail-1 mutant, while all aphids were dead on the wild-type parent (Fig. 2A). Potato aphids have a viviparous mode of reproduction, and progeny were counted on a daily basis to evaluate aphid fecundity. The average number of progeny per day per aphid on the jail-1 mutant was not significantly different from the number of progeny on cv. CM $(P>0.001)$ (Fig. 2B). Similarly, the total number of progeny per aphid was not significantly different $(P>0.05)$ on jail-1 mutant compared with cv. CM (Fig. 2C).

\section{Genetic cross and identification of homozygous $\mathrm{Mi}$-1 jail plants.}

The tomato jail-1 mutant does not contain the Mi-1 gene. To evaluate whether Coil-dependent signaling plays a role in Mi-1-mediated aphid resistance, we introduced $M i-1$ in the jail-1 mutant background. Genetic crosses were performed between VFN tomato and the jail-1 mutant, F1 plants were allowed to self, and the $\mathrm{F} 2$ population was evaluated for sensitivity to MeJA and the presence of a deletion in the Coil gene. F2

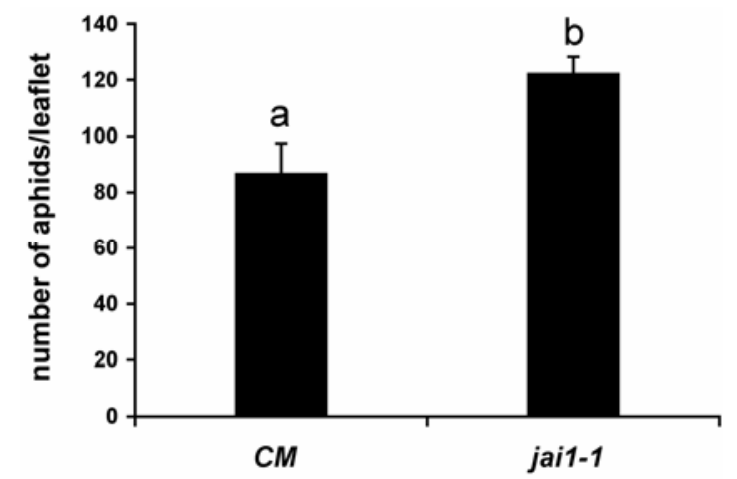

Fig. 1. A choice assay on tomato jail-1 mutant and wild-type parent cv. Castlemart (CM) plants. Six-week-old tomato plants were exposed to potato aphids in an insect cage in a greenhouse. Plants were evaluated 14 days after exposure to aphids. Ten plants per genotype were used. The experiment was performed twice with similar results. Data representing means from one experiment are presented. Error bars indicate \pm standard error. Bars with different letters denote a significant difference at $P<0.05$. 
plants insensitive to MeJA were further evaluated for the presence of the $M i-1$ gene, using the linked marker REX-1 (data not shown) (Williamson et al. 1994). REX-1 is a codominant marker that allows for the identification of the $S$. peruvianum introgressed region spanning $M i-1$. Plants homozygous for the $M i-1$ locus and for the coil deletion were used for further evaluations.

\section{Expression of JA regulated wound-induced genes in $\mathrm{Mi}$-1 jail plants}

To assess the integrity of the JA signaling pathway in the homozygous $M i-1$ jail plants (referred to as VFN $\times$ jail -1 ), the

\section{A}

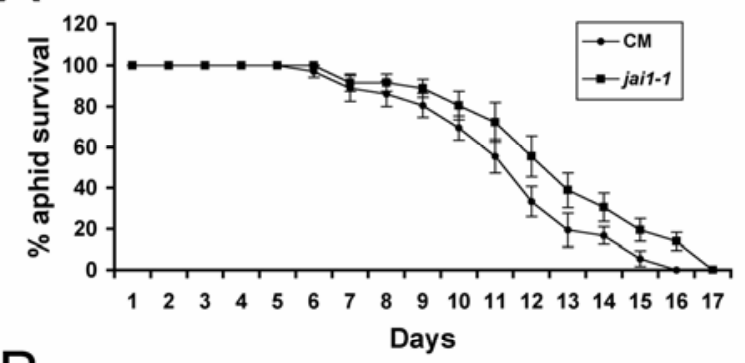

B

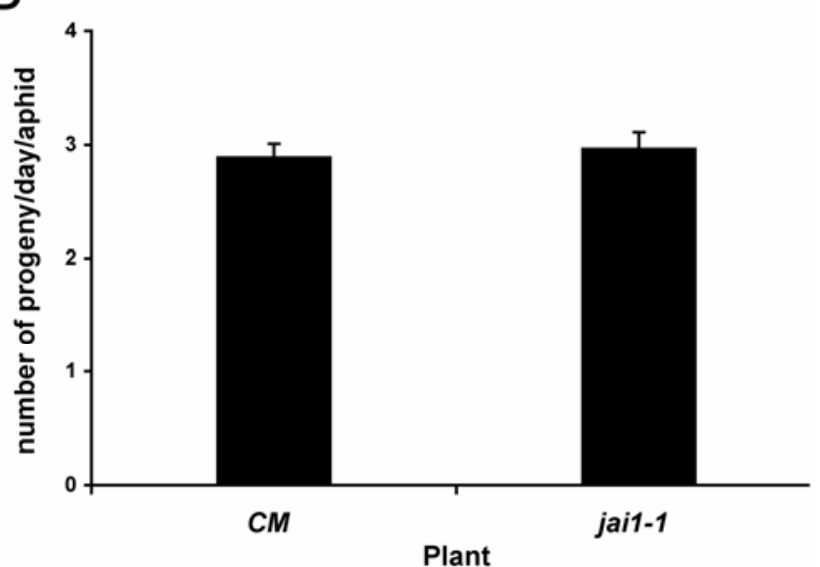

C

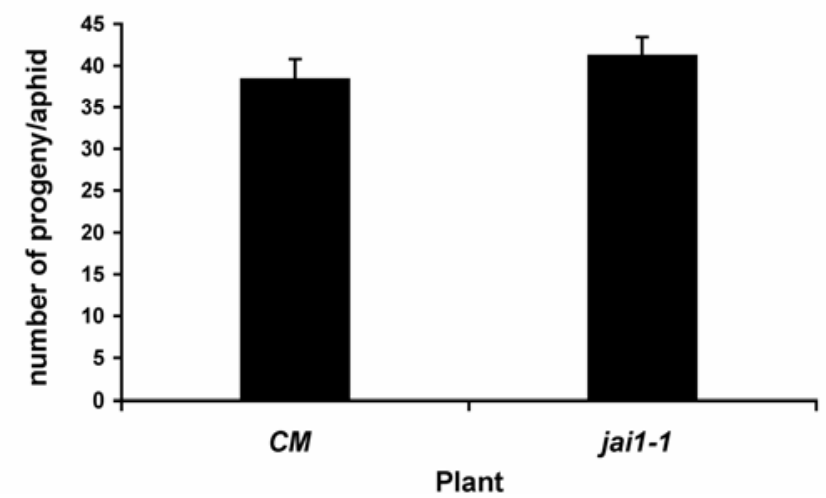

Fig. 2. Aphid survival and fecundity on tomato wild-type cv. Castlemart (CM) and jail-1 mutant plants. Four-week-old tomato plants were used in no-choice assays. A single leaflet per plant was infested with four one-dayold aphids using clip cages, and aphid survival and fecundity were monitored daily. Nine plants of each genotype were used. Error bars indicate \pm standard error. The experiment was performed twice with similar results. Data representing one experiment are presented. A, Daily aphid survival. Statistical analysis was performed on arcsin-transformed data, and significant differences at $P<0.05$ are indicated by an (*). B, Daily aphid reproduction. $\mathbf{C}$, Aphid reproduction. Bars with similar letters denote no significant difference at $P>0.001$. expression of allene oxide synthase 1 (AOS1), an octadecanoid pathway enzyme encoding a cytochrome P450, and Pinl were evaluated (Howe et al. 2000). AOS1 and Pinl are induced after wounding early and late, respectively (Ryan 2000). Tomato leaflets either wounded or exposed to potato aphids were used in these experiments. In VFN leaflets, AOS1 transcripts accumulated to high levels at $4 \mathrm{~h}$ after wounding and were absent at $24 \mathrm{~h}$ (Fig. 3). Similarly, AOS1 transcripts were expressed transiently at $4 \mathrm{~h}$ but to a lesser extent in both jail-1 mutant and VFN $\times$ jail-1 leaflets (Fig. 3). In contrast, AOS 1 transcripts were not detected at 6 and $12 \mathrm{~h}$ after aphid feeding on any of the three genotypes tested (Fig. 3). In VFN leaflets, Pin1 transcripts accumulated to very high levels at both 4 and $24 \mathrm{~h}$ after wounding (Fig. 3). Pinl transcripts accumulated in VFN leaflets 6 and $12 \mathrm{~h}$ after aphid feeding, confirming an earlier report (Martinez de Ilarduya et al. 2003). In contrast, no Pinl transcripts were detected in either jail-1 or VFN $\times$ jail-1 leaflets after wounding or aphid feeding, indicating that the JA signaling pathway is compromised in these plants (Fig. 3).

\section{Potato aphid survival on $\mathrm{Mi}$-1 jail plants.}

Both choice and no-choice assays with potato aphids were used to evaluate $M i-1$ plants compromised in the JA signaling pathway. In choice assays, in which insects were allowed to choose among jail-1, VFN, or VFN $\times$ jail-1 plants, significantly higher numbers of aphids were present on jail-1 plants compared with VFN $(P<0.001)$ and VFN $\times$ jail $1-1$ plants $(P<$ 0.001) (Fig. 4A and B). There was no significant difference between number of aphids on VFN $\times$ jail-1 compared with VFN plants $(P>0.001)$, indicating that the Coil-dependent signaling pathway is not required for $\mathrm{Mi}$ - 1 -mediated aphid resistance (Fig. 4A and 4B). To further confirm these results, a no-choice assay was performed with one-day-old adult aphids. The no-choice assay confirmed the results obtained in the choice assay. The number of aphids on VFN $\times$ jail-1 plants was similar to the number of aphids on VFN plants $(P>0.001)$ but was significantly higher than the number of aphids on jail1 mutant plants $(P<0.001)$ (Fig. 4C). Similarly, there was a significantly lower number of aphids on VFN compared with jail-1 plants $(P<0.001)$ (Fig. 4C).

\section{DISCUSSION}

Coil does not play a role in $\mathrm{Mi}$-1-mediated aphid resistance.

Our results do not support a role for a Coil-dependent signaling pathway in $\mathrm{Mi}-1-$ mediated resistance to potato aphids. In spite of this finding, transcripts of both defense-related proteins Pin1 (Fig. 3) and Pin2 (Martinez de Ilarduya et al. 2003) are induced early after aphid feeding on resistant plants, suggesting that tomato JA-dependent defenses are activated in the incompatible interaction. In general, faster and higher levels of transcript accumulation is associated with incompatible interactions (Tao et al. 2003). Since temporal expression of Pinl and Pin2 was similar in both compatible and incompatible interactions, JA-dependent plant defense could contribute to basal defense rather than to Mi-1-mediated defense (Martinez de Ilarduya et al. 2003).

Mi-1-mediated aphid and nematode resistance requires the SA signaling pathway (Branch et al. 2004; Li et al. 2006). Introduction of $N a h G$ in the $M i-1$ background abolished aphid resistance in leaves, allowing aphids to multiply on $\mathrm{Mi}-1$ plants (Li et al. 2006). Application of benzothiadiazole, a SA analog, was able to rescue the susceptible phenotype in $\mathrm{Mi}-1 \mathrm{NahG}$ plants, indicating that SA is required for $M i-1-$ mediated aphid defense (Li et al. 2006). In this article, it was demonstrated that 
Mi-1-mediated aphid resistance is unaffected by the absence of a functional JA pathway, indicating that JA is not required for resistance. The requirement of SA but not JA in Mi-1mediated aphid defense in tomato demonstrates that gene-forgene resistance against aphids functions through similar defense signaling pathways as to most plant pathogens (Kaloshian 2004; Kaloshian and Walling 2005).

\section{Gene regulation in tomato with the jail mutation.}

$A O S$ genes are induced transiently after wounding (Howe et al. 2000; Sivasankar et al. 2000) and application of MeJA (Li et al. 2004; Sivasankar et al. 2000). The lack of observed AOS1 expression after aphid feeding on tomato genotypes is likely due to timing of tissue collection or the magnitude of the response. In a previous study, AOS1 transcripts accumulated locally to high levels 1 and $2 \mathrm{~h}$ after wounding and decreased to nondetectable levels by $8 \mathrm{~h}$ (Lee and Howe 2003). Our aphid infestation timecourse ( 6 and $12 \mathrm{~h}$ after infestation) was selected to allow for the detection of Pinl transcripts (Martinez de Ilarduya et al. 2003). Tissue collection at earlier timepoints might have allowed detection of AOS1 transcripts. Alternatively, the accumulation of AOS1 transcripts by aphid feeding is lower than our detection limit.

A decrease in AOS1 transcript levels was observed after wounding in jail-1 and VFN $\times$ jail-1 plants compared with VFN, reflecting a similar pattern as that shown for AOS 2 in response to exogenous MeJA treatment of jail-1 compared with wild-type plants ( $\mathrm{Li}$ et al. 2004) and indicating that woundinducible AOS1 transcript accumulation is also partly Coil dependent.

The lack of Pinl transcript accumulation after wounding in jail-1 and VFN $\times$ jail-1 plants confirmed earlier findings that expression of wound-induced proteinase inhibitors is Coil dependent (Li et al. 2001). Similarly, the lack of Pinl transcript accumulation in aphid-infested leaf tissue indicated that the aphid-induced Pinl expression is also regulated by Coil (Fig. 3). Pin1 transcript accumulation is most likely due to wounding caused by aphid feeding. Although aphid stylets penetrate host tissue mainly intercellularly to reach the sieve element where they feed, intracellular penetration also occurs. In fact, $27 \%$ of the probes by potato aphids penetrate tomato tissue intracellularly, which may explain the induction of wound responses (Kaloshian et al. 2000). However, the JA-dependent wound signaling pathway did not contribute to aphid defense, since jail-1 mutant and wild-type tomato plants supported similar levels of aphid fecundity and survival.
The role of JA-regulated signaling

in basal defense to aphids.

A differential role for JA is indicated depending on the aphid assay used. A small but significantly different increase in colonization of aphids was observed on the jail-1 mutant as compared with the wild-type parent in the choice assay (Fig. 1). Since no significant differences in aphid survival and fecundity was observed between jail-1 mutant and wild-type plants, the larger number of aphids observed on jail-1 plants could be due to changes in the oxylipin signature of this mutant that could enhance aphid attraction to jail-1 plants.

Eliminating insect choice between genotypes and movement between plants resulted in no difference in aphid survival or fecundity on jail-1 plants as compared with wild type, indicating no role for Coil-dependent basal plant defense against potato aphids. In several plant species, stronger activation of SAthan JA-regulated genes by aphid feeding have been observed (Martinez de Ilarduya et al. 2003; Moran and Thompson 2001; Zhu-Salzman et al. 2004). Since cross talk exists between SA and JA signaling pathways, this and other information have lead to speculations that aphids might manipulate plant defenses by activating ineffective SA responses to suppress effective JA responses (Thompson and Goggin 2006; Zhu-Salzman et al. 2004). Our results indicate that tomato-potato aphid interaction does not follow this model. Although aphids activate both SA and JA responses, eliminating the JA response did not alter aphid defense.

In Arabidopsis, the role of the JA-regulated pathway in aphid defense is not well defined. For example, the Arabidopsis coil mutation had no effect on Myzus persicae population growth (Mewis et al. 2005). In contrast, the same researchers reported more rapid growth of the specialist aphid Brevicoryne brassicae on the coil mutant compared with the wild-type parent (Mewis et al. 2005). In addition, the Arabidopsis cevl mutant, which expresses JA-dependent responses constitutively, supported low Myzus persicae population growth, supporting a role for the JA-regulated defense in Arabidopsis to aphids (Ellis et al. 2002). Since the cevl mutation has pleiotropic effects, it is difficult to reach conclusions using this mutant (Ellis and Turner 2001). These results indicate that, in Arabidopsis, the role of the JA-regulated pathway in basal resistance to aphids remains controversial and suggests that different plant species may involve distinct defense signaling pathways (Kaloshian and Walling 2005; Thompson and Goggin 2006).

Exogenous application of MeJA reduced greenbug aphid (Schizaphis graminum) attraction to sorghum seedlings (Zhu-

Wounded

Aphid infested

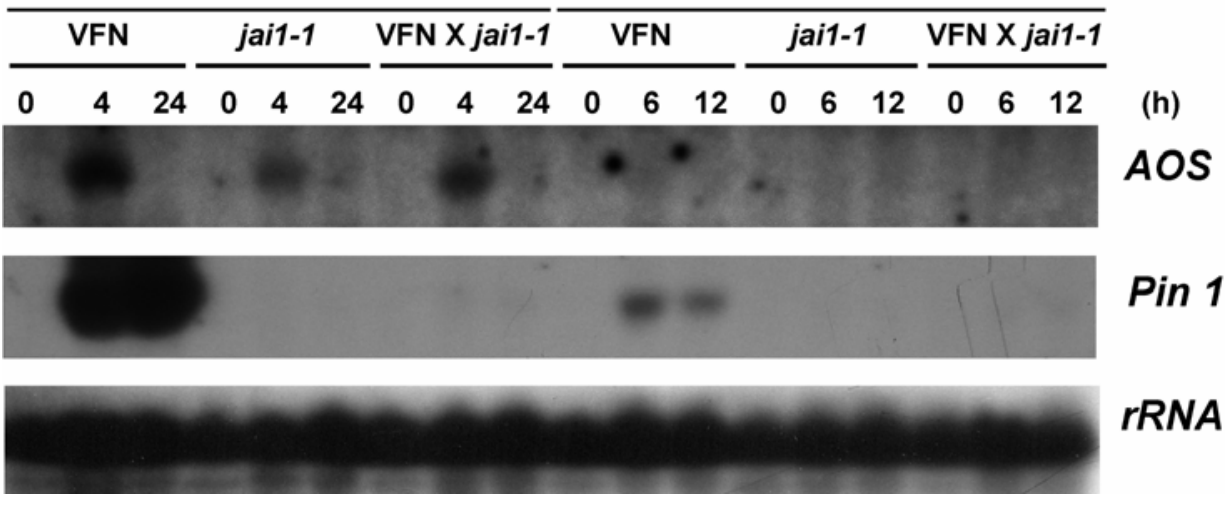

Fig. 3. Transcript accumulation in leaflets of tomato cv. VFN (Mi-1/Mi-1 Jail/Jail), jail-1 (mi/mi jail/jail), and VFN $\times$ jail-1 (Mi-1/Mi-1 jail/jail) after wounding or potato aphid infestations. Leaflets of seven-week-old tomato plants were either wounded or infested with potato aphids. Wound samples were collected at 0,4 , and $24 \mathrm{~h}$ after wounding. Aphid-infested samples were collected 0,6 , and $12 \mathrm{~h}$ after infestations. Total RNA was isolated, and the RNA blot was hybridized to probes for LeAOS1 (AOS), proteinase inhibitor I (Pin1), and 18S rRNA as a loading control. 

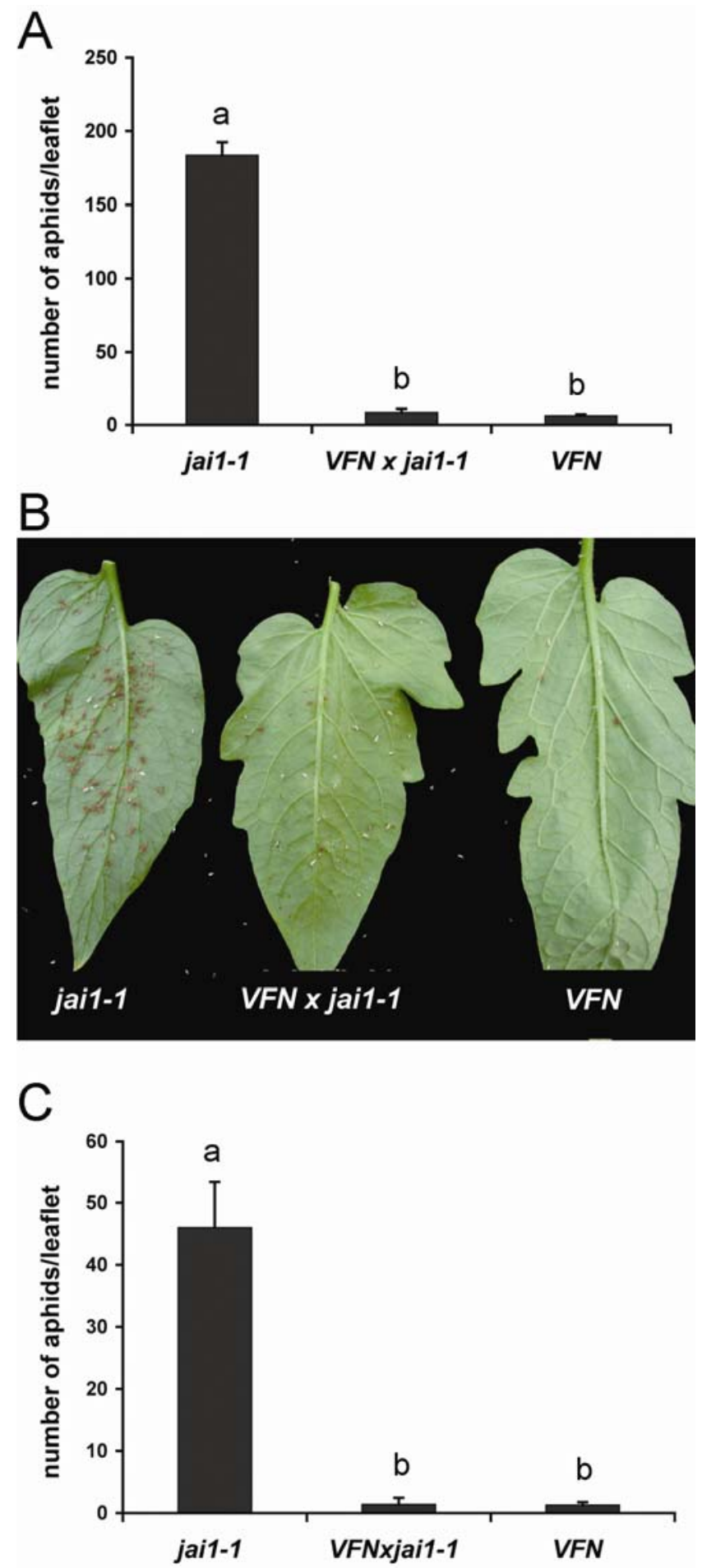

Fig. 4. Aphid assays of tomato cv. VFN (Mi-1/Mi-1 Jail/Jail), jail-1 (mi/mi jail/jail), and VFN × jail-1 (Mi-1/Mi-1 jail/jail) plants. A, Aphid choice assay with seven-week-old tomato plants in an insect cage in a greenhouse. Plants were evaluated 14 days after exposure to aphids. Eight plants per genotype were used. The experiment was performed twice with similar results. Data representing means from one experiment are presented. Error bars indicate \pm standard error. Bars with different letters denote a significant difference at $P<0.001$. B, Phenotype of tomato leaflets infested with potato aphids in the choice assays. C, Aphid survival in a nochoice assay. Individual leaflets of seven-week-old tomato were infested with four one-day-old aphids using leaf cages. Four leaflets per plant and eight plants of each genotype were infested. The number of aphids was evaluated 14 days after aphid infestation. The experiment was performed one time. Error bars indicate \pm standard error. Bars with different letters denote a significant difference at $P<0.001$.
Salzman et al. 2004) and Myzus persicae growth on Arabidopsis (Ellis et al. 2002). Similarly, exogenous JA application reduced potato aphid host preference, survival, and fecundity on susceptible tomato plants lacking $\mathrm{Mi}-\mathrm{l}$ (Cooper and Goggin 2005). However, this negative effect was not seen on resistant Mi-1 tomato plants (Cooper and Goggin 2005). These results suggest that artificial augmentation of the JA pathway enhances aphid basal resistance and this effect is not exhibited in resistant plants. Alternatively, the exogenous application of JA might affect the pattern of other oxylipins that might be involved in aphid resistance (Avdiushko et al. 1995; Kohlmann et al. 1999). Recently, a role for the JA precursor 12-oxo-phytodienoic acid independent of JA was described (Stintzi et al. 2001; Taki et al. 2005). It is, therefore, possible that a Coil-independent signaling pathway mediated by other oxilipins might play a role in aphid defense.

\section{MATERIALS AND METHODS}

Plant materials and growth conditions.

The tomato cultivars and mutants used in this study were: VFN (Mi-1/Mi-1), UC82B ( $\mathrm{mi} / \mathrm{mi}), \mathrm{CM}(\mathrm{mi} / \mathrm{mi})$, and jail-1 mutant in CM background ( $\mathrm{Li}$ et al. 2004). Seeds were sown in seedling trays filled with organic planting mix (Sun Gro Horticulture, Bellevue, WA, U.S.A.) or were sown and grown directly in Jiffy peat pots (Jiffy Product of America, Inc., Batavia, IL, U.S.A.). To promote uniform germination, seedling trays were maintained in an enclosed greenhouse structure with misters. After germination, seedlings were grown in a pesticide-free greenhouse inside large plant cages to avoid insect infestations, with temperatures ranging from 22 to $26^{\circ} \mathrm{C}$. Two to three weeks after germination, seedlings were transplanted into plastic pots $(10 \mathrm{~cm}$ in diameter, $17 \mathrm{~cm}$ deep) filled with a $1: 1$ proportion of University of California mix II and sand. All seedlings were supplemented with Osmocote (17-6-10) (Sierra Chemical Company, Milpitas, CA, U.S.A.) and were fertilized biweekly with Tomato MiracleGro (18-18-21) (Stern's MiracleGro Products, Port Washington, NY, U.S.A.).

\section{Aphid rearing.}

A colony of a parthenogenetic $M i$-1-avirulent potato aphid, Macrosiphum euphorbiae, was maintained on susceptible tomato cv. UC82B plants inside an insect cage in a pesticide-free greenhouse at 22 to $26^{\circ} \mathrm{C}$. Artificial lighting was supplemented during the winter season to increase day length to a $16 \mathrm{~h}$ photoperiod.

To obtain one-day-old adult aphids, adult apterous (wingless) aphids were transferred to tomato seedlings and were allowed to lay progeny. Seedlings were maintained in an insect cage in a pesticide-free greenhouse. About $24 \mathrm{~h}$ after aphid infestation, the adult aphids were removed, using a Pasteur pipette hooked to an aspirator, and the first instars were allowed to develop to maturity.

\section{Chemical screen of seedlings with MeJA.}

Seedlings were screened for sensitivity to MeJA according to Li and associates (2004). Briefly, surface-sterilized tomato seeds were germinated on filter paper until the roots were $2 \mathrm{~cm}$ in length. The germinated seedlings were exposed to MeJA by saturating the filter paper with $1 \mathrm{mM}$ MeJA. Approximately 1 day later, MeJA insensitive seedlings were selected and transplanted.

\section{Genetic crosses.}

Genetic crosses were performed between VFN and jail-1 mutant. Since mutation in the Coil gene in tomato results in sterility due to maternally-controlled improper seed maturation 
(Li et al. 2004), we used VFN as the female parent and the jail-1 mutant as the source of pollen. To obtain homozygous jail-1 pollen, seedlings segregating for the locus were evaluated for MeJA insensitivity and the presence of the coil deletion as described above.

\section{Molecular genotyping.}

Rex-1 and Coil loci were amplified using PCR. The Rex-1 primers and amplification conditions are described by Williamson and associates (1994), and the tomato Coil primers and amplification conditions are described by $\mathrm{Li}$ and associates (2004).

\section{Timecourse aphid infestations.}

For timecourse transcript analyses, 25 apterous potato aphid adults and nymphs were caged onto individual leaflets from the fourth or fifth leaves of 7-week-old tomato plants. The leaf cages used in this experiment were as described by Li and associates (2006). Three cages were used per plant, and two plants were used for each timepoint/genotype combination. To collect leaf tissue, cages were removed and leaflets were sprayed with $1 \%$ sodium dodecyl sulfate (SDS) to force aphids to withdraw their stylets. The aphids were then carefully removed with a paintbrush. Tomato leaflets were excised using a razor blade, were immediately frozen in liquid nitrogen, and were stored at $-80^{\circ} \mathrm{C}$.

\section{Aphid choice bioassay.}

In choice assays, test plants were moved into large cages inside a pesticide-free greenhouse. Several pots of aphidinfested, four-week-old susceptible tomato seedlings, 20 seedlings per pot (12 cm diameter, $10 \mathrm{~cm}$ deep), were distributed within a cage. Since the aphid source plants were smaller in size compared with the screened plants, pots containing the aphid source were raised to the mid level of the screened plants to allow easy access of aphids to the experimental plants. At 14 days after aphid exposure, aphids on the mostinfested three leaflets of each plant were counted. The experiment was performed twice.

\section{Aphid no-choice bioassay.}

Two distinct no-choice aphid bioassays were performed. In the first no-choice assay, four one-day-old adult apterous aphids were caged onto the abaxial leaf surface of a single leaflet of a four-week-old tomato using a clip cage. Nine replicates of each genotype were used. Plants were placed randomly on a bench in a pesticide-free greenhouse maintained at 22 to $26^{\circ} \mathrm{C}$. Aphid survival was monitored and recorded on a daily basis. To evaluate aphid fecundity, first instar progeny were counted and removed, using an aspirator on a daily basis. The experiment was performed twice.

In the second no-choice bioassay in which $\mathrm{Mi}-\mathrm{l}$ tomato plants were involved, four one-day-old adult apterous aphids were caged onto individual leaflets of seven-week-old tomato, as described for the timecourse infestation. Plants were placed randomly on a bench in a pesticide-free greenhouse maintained at 22 to $26^{\circ} \mathrm{C}$. Plants were evaluated by counting the number of aphids on each caged leaflet 14 days after aphid infestations.

\section{Statistical analyses.}

Data having three or more treatments were analyzed using analysis of variance (ANOVA) on raw data using the statistical package SAS (v9.1 for Windows, SAS Institute, Inc., Cary, NC, U.S.A.). Pairwise comparisons were performed using Fisher's probable least-squares difference. Differences in means for two genotypes were performed, using a two-sample $t$ test also using the statistical package SAS. Data in percentage values were arcsin-transformed before analysis to correct for normality and differences in variance between treatments. Daily aphid survival across days was analyzed using repeated measures ANOVA to account for the correlations over time in the consecutive data.

\section{RNA isolation and gel blot analysis.}

Total RNA was isolated as described previously (Martinez de Ilarduya et al. 2001). Total RNA (15 $\mu \mathrm{g}$ per lane) was separated on a $1.2 \%$ agarose/formaldehyde gel and was transferred to a nylon membrane (Osmonics, Inc., Minnetonka, MN, U.S.A.) in $10 \times \mathrm{SSC}(1 \times \mathrm{SSC}$ is $0.15 \mathrm{M} \mathrm{NaCl}$ plus $0.015 \mathrm{M}$ sodium citrate, $\mathrm{pH}$ 7.0). The gel was stained with ethidium bromide to assure equal loading per lane. A Pinl cDNA clone was kindly provided by C. A. Ryan (Washington State University, Pullman, WA, U.S.A.). The tomato expressed sequence tag clone cLEC9C14 was used as probe for AOS1. A soybean rRNA clone was used as a control of equal transfer between lanes. DNA probes were labeled with ${ }^{32} \mathrm{P}-\alpha-\mathrm{dCTP}$, using the Redi-prime labeling kit (Amersham, Arlington Heights, IL, U.S.A.) according to the manufacturer's instructions. RNA blots were prehybridized for $2 \mathrm{~h}$ at $42^{\circ} \mathrm{C}$ and were hybridized for $16 \mathrm{~h}$ at $42^{\circ} \mathrm{C}$, as described by Martinez de Ilarduya and associates (2001). The final blot wash was in $0.5 \times \mathrm{SSC}, 0.1 \%$ SDS at $65^{\circ} \mathrm{C}$ for $30 \mathrm{~min}$. Hybridization results were visualized by autoradiography using Kodak XAR-5 film. Probes were stripped by pouring boiling $0.5 \%$ SDS on the membrane. Before reuse, the membrane was checked for complete removal of probe.

\section{Wound treatment.}

Tomato leaflets were wounded by crushing with a pair of pliers on two locations on the midrib. Three leaves per plant were wounded, two plants were used for each timepoint, and tissue was pooled. Unwounded leaflets from the two plants were sampled at the beginning of the experiment. Wounded leaflets were harvested at 4 and $12 \mathrm{~h}$ after wounding. Sampled leaf tissue was immediately frozen in liquid nitrogen and stored at $-80^{\circ} \mathrm{C}$.

\section{ACKNOWLEDGMENTS}

We thank Q. Li for performing the genetic cross and D. Sergeant for technical help. We also thank G. Howe, Michigan State University, for the jail-1 and CM seeds. We are also grateful to S. Edwards for photography and J. Smith-Becker for reading the manuscript. This research was supported by grants from the University of California Agricultural Experiment Station and the National Science Foundation (IOB-0543937) to I. Kaloshian.

\section{LITERAURE CITED}

Avdiushko, S., Croft, K. P., Brown, G. C., Jackson, D. M., HamiltonKemp, T. R., and Hildebrand, D. 1995. Effect of volatile methyl jasmonate on the oxylipin pathway in tobacco, cucumber, and Arabidopsis. Plant Physiol. 109:1227-1230.

Bai, C., Sen, P., Hofmann, K., Ma, L., Goebl, M., Harper, J. W., and Elledge, S. J. 1996. SKP1 connects cell cycle regulators to the ubiquitin proteolysis machinery through a novel motif, the F-box. Cell 86:263-274.

Branch, C., Hwang, C.-F., Navarre, D. A., and Williamson, V. M. 2004. Salicylic acid is part of the $M i$-1-mediated defense response to rootknot nematode in tomato. Mol Plant-Microbe Interact 17:351-356.

Chao, W., Gu, Y., Pautot, V., Bray, E., and Walling, L. 1999. Leucine aminopeptidase RNAs, proteins, and activities increase in response to water deficit, salinity, and the wound signals systemin, methyl jasmonate, and abscisic acid. Plant Physiol. 120:979-992.

Cooper, W. R., and Goggin, F. L. 2005. Effects of jasmonate-induced defenses in tomato on the potato aphid, Macrosiphum euphorbiae. Entomol. Exp. Appl. 115:107-115.

Creelman, R. A., and Mullet, J. E. 1997. Biosynthesis and action of jasmonates in plants. Annu. Rev. Plant Physiol. Plant Mol. Biol. 48:355-381. 
Dropkin, V. H. 1969. Cellular responses of plants to nematode infections. Annu. Rev. Phytopathol. 7:101-122.

Ellis, C., and Turner, J. G. 2001. The Arabidopsis mutant cev1 has constitutively active jasmonate and ethylene signal pathways and enhanced resistance to pathogens. Plant Cell 13:1025-1033.

Ellis, C., Karafyllidis, I., and Turner, J. G. 2002. Constitutive activation of jasmonate signaling in an Arabidopsis mutant correlates with enhanced resistance to Erysiphae cichoracearum, Pseudomonas syringae, and Myzus persicae. Mol. Plant-Microbe Interact. 15:1025-1030.

Farmer, E. E., and Ryan, C. A. 1992. Octadecanoid precursors of jasmonic acid activate the synthesis of wound-inducible proteinase inhibitors. Plant Cell 4:129-134.

Flor, H. H. 1971. Current status of the gene-for-gene concept. Annu. Rev. Phytopathol. 9:275-296.

Hammond-Kosack, K. E., and Parker, J. E. 2003. Deciphering plant-pathogen communication: Fresh perspectives for molecular resistance breeding. Curr. Opin. Biotechnol. 14:177-193.

Howe, G. A., Lee, G. I., Itoh, A., Li, L., and DeRocher, A. E. 2000. Cytochrome P450-dependent metabolism of oxylipins in tomato. Cloning and expression of allene oxide synthase and fatty acid hydroperoxide lyase. Plant Physiol. 123:711-724.

Kaloshian, I. 2004. Gene-for-gene disease resistance: Bridging insect pest and pathogen defense. J. Chem. Ecol. 30:2421-2439.

Kaloshian, I., and Walling, L. L. 2005. Hemipterans as plant pathogens. Annu. Rev. Phytopathol. 43:491-521.

Kaloshian, I., Lange, W. H., and Williamson, V. M. 1995. An aphid resistance locus is tightly linked to the nematode resistance gene, $\mathrm{Mi}$, in tomato. Proc. Natl. Acad. Sci. U.S.A. 92:622-625.

Kaloshian, I., Kinsey, M. G., Ullman, D. E., and Williamson, V. M. 1997. The impact of Meul-mediated resistance in tomato on longevity, fecundity and behavior of the potato aphid, Macrosiphum euphorbiae. Entomol. Exp. Appl. 83:181-187.

Kaloshian, I., Kinsey, M. G., Williamson, V. M., and Ullman, D. E. 2000. Mi-mediated resistance against the potato aphid Macrosiphum euphorbiae (Hemiptera: Aphididae) limits sieve element ingestion. Environ. Entomol. 29:690-695.

Kohlmann, M., Bachmann, A., Weichert, H., Kolbe, A., Balkenhohl, T., Wasternack, C., and Feussner, I. 1999. Formation of lipoxygenase-pathway-derived aldehydes in barley leaves upon methyl jasmonate treatment. Eur. J. Biochem. 260:885-895.

Lee, G. I., and Howe, G. A. 2003. The tomato mutant spr1 is defective in systemin perception and the production of a systemic wound signal for defense gene expression. Plant J. 33:567-576.

Li, L., Li, C. Y., and Howe, G. A. 2001. Genetic analysis of wound signaling in tomato. Evidence for a dual role of jasmonic acid in defense and female fertility. Plant Physiol. 127:1414-1417.

Li, L., Zhao, Y., McCaig, B. C., Wingerd, B. A., Wang, J., Whalon, M. E., Pichersky, E., and Howe, G. A. 2004. The tomato homolog of CORONATINE-INSENSITIVE1 is required for the maternal control of seed maturation, jasmonate-signaled defense responses, and glandular trichome development. Plant Cell 16:126-143.

Li, Q., Xie, Q., Smith-Becker, J., Navarre, D., and Kaloshian, I. 2006. Mi1-mediated aphid resistance involves salicylic acid and mitogen-activated protein kinase signaling pathways. Mol. Plant-Microbe Interact. 19:655-664.

Martin, G. B., Bogdanove, A. J., and Sessa, G. 2003. Understanding the functions of plant disease resistance proteins. Annu. Rev. Plant Biol. 54:23-61.

Martinez de Ilarduya, O., and Kaloshian, I. 2001. Mi-1.2 transcripts accumulate ubiquitously in root-knot nematode resistant Lycopersicon esculentum. J. Nematol. 33:116-120.

Martinez de Ilarduya, O., Moore , A. E., and Kaloshian, I. 2001. The tomato Rmel locus is required for $M i-1$-mediated resistance to root-knot nematodes and the potato aphid. Plant J. 27:417-425.

Martinez de Ilarduya, O., Xie, Q.-G., and Kaloshian, I. 2003. Aphid- induced defense responses in $\mathrm{Mi}$-1-mediated compatible and incompatible tomato interactions. Mol. Plant-Microbe Interact. 16:699-708.

Martinez de Ilarduya, O., Nombela, G., Hwang, C.-F., Williamson, V. M., Muñiz, M., and Kaloshian, I. 2004. Rmel is necessary for Mi-1-mediated resistance and acts early in the resistance pathway. Mol. PlantMicrobe Interact. 17:55-61.

Mewis, I., Appel, H. M., Hom, A., Raina, R., and Schultz, J. C. 2005. Major signaling pathways modulate Arabidopsis glucosinolate accumulation and response to both phloem-feeding and chewing insects. Plant Physiol. 138:1149-1162.

Milligan, S. B., Bodeau, J., Yaghoobi, J., Kaloshian, I., Zabel, P., and Williamson, V. M. 1998. The root-knot nematode resistance gene $M i$ from tomato is a member of leucine zipper, nucleotide binding, leucinerich repeat family of plant genes. Plant Cell 10:1307-1319.

Moran, P. J., and Thompson, G. A. 2001. Molecular responses to aphid feeding in Arabidopsis in relation to plant defense pathways. Plant Physiol. 125:1074-1085.

Nombela, G., Williamson, V. M., and Muñiz, M. 2003. The root-knot nematode resistance gene $\mathrm{Mi}-1.2$ of tomato is responsible for resistance against the whitefly Bemisia tabaci. Mol. Plant-Microbe Interact. 16:645-649.

Pascual, S., Avilés, M., Nombela, G., Muñiz, M., and Beitia, F. 2000. Development of Bemisia tabaci (biotype Q) on tomato cultivars with/without the $M i$ gene. Med. Fac. Landbouww. Univ. Gent. 65/2a:291-292.

Ryan, C. A. 2000. The systemin signaling pathway: Differential activation of plant defensive genes. Biochim. Biophys. Acta 1477:112-121.

Schenk, P., Kazan, K., Wilson, I., Anderson, J., Richmond, T., Somerville, S., and Manners, J. 2000. Coordinated plant defense responses in Arabidopsis revealed by microarray analysis. Proc. Natl. Acad. Sci. U.S.A. 97:11655-11660.

Sivasankar, S., Sheldrick, B., and Rothstein, S. J. 2000. Expression of allene oxide synthase determines defense gene activation in tomato. Plant Physiol. 122:1335-1342.

Smith, P. G. 1944. Embryo culture of a tomato species hybrid. Proc. Am. Soc. Hortic. Sci. 44:413-416.

Stintzi, A., Weber, H., Reymond, P., Browse, J., and Farmer, E. E. 2001. Plant defense in the absence of jasmonic acid: The role of cyclopentenones. Proc. Natl. Acad. Sci. U.S.A. 98:12837-12842.

Taki, N., Sasaki-Sekimoto, Y., Obayashi, T., Kikuta, A., Kobayashi, K. Ainai, T., Yagi, K., Sakurai, N., Suzuki, H., Masuda, T., Takamiya, K., Shibata, D., Kobayashi, Y., and Ohta, H. 2005. 12-oxo-phytodienoic acid triggers expression of a distinct set of genes and plays a role in wound-induced gene expression in Arabidopsis. Plant Physiol. 139:1268-1283.

Tao, Y., Xie, Z. Y., Chen, W. Q., Glazebrook, J., Chang, H. S., Han, B., Zhu, T., Zou, G. Z., and Katagiri, F. 2003. Quantitative nature of Arabidopsis responses during compatible and incompatible interactions with the bacterial pathogen Pseudomonas syringae. Plant Cell 15:317-330.

Thompson, G. A., and Goggin, F. L. 2006. Transcriptomics and functional genomics of plant defence induction by phloem-feeding insects. J. Exp. Bot. 57:755-766.

Turner, J. G., Ellis, C., and Devoto, A. 2002. The jasmonate signal pathway. Plant Cell 14 Suppl:S153-64.

van Kan, J., Cozijnsen, T., Danhash, N., and de Wit, P. 1995. Induction of tomato stress protein mRNAs by ethephon, 2,6-dichloroisonicotinic acid and salicylate. Plant Mol. Biol. 27:1205-1213.

Williamson, V. M., Ho, J.-Y., Wu, F. F., Miller, N., and Kaloshian, I. 1994. A PCR-based marker tightly linked to the nematode resistance gene, $M i$, in tomato. Theor. Appl. Genet. 87:757-763.

Xie, D. X., Feys, B. F., James, S., Nieto-Rostro, M., and Turner, J. G. 1998. COI1: An Arabidopsis gene required for jasmonate-regulated defense and fertility. Science 280:1091-1094.

Zhu-Salzman, K., Salzman, R. A., Ahn, J. E., and Koiwa, H. 2004. Transcriptional regulation of sorghum defense determinants against a phloem-feeding aphid. Plant Physiol. 134:420-431. 